\title{
Metabolism of phosphatidylcholine and its implications for lipid acyl chain composition in Saccharomyces cerevisiae
}

\author{
Anton I.P.M. de Kroon \\ Department Biochemistry of Membranes, Bijvoet Institute and Institute of Biomembranes, Utrecht University, Padualaan 8, 3584 CH Utrecht, The Netherlands
}

Received 31 March 2006; received in revised form 28 July 2006; accepted 31 July 2006

Available online 2 August 2006

\begin{abstract}
Phosphatidylcholine (PC) is a very abundant membrane lipid in most eukaryotes including the model organism Saccharomyces cerevisiae. Consequently, the molecular species profile of PC, i.e. the ensemble of PC molecules with acyl chains differing in number of carbon atoms and double bonds, is important in determining the physical properties of eukaryotic membranes, and should be tightly regulated. In this review current insights in the contributions of biosynthesis, turnover, and remodeling by acyl chain exchange to the maintenance of PC homeostasis at the level of the molecular species in yeast are summarized. In addition, the phospholipid class-specific changes in membrane acyl chain composition induced by PC depletion are discussed, which identify PC as key player in a novel regulatory mechanism balancing the proportions of bilayer and nonbilayer lipids in yeast.
\end{abstract}

(C) 2006 Elsevier B.V. All rights reserved.

Keywords: Phosphatidylcholine; Yeast; Lipid metabolism; Acyl chain remodeling; Lipid composition; Non-bilayer lipid

\section{Introduction}

The glycerophospholipid phosphatidylcholine (PC) is the major lipid constituent of the membranes of most eukaryotes including Saccharomyces cerevisiae, comprising up to $50 \%$ of total phospholipids [1]. Because of its overall cylindrical molecular shape, PC spontaneously organizes into bilayers [2], making it ideally suited to serve as the bulk structural element of biological membranes. In addition to its function as a membrane building block preserving membrane barrier function, $\mathrm{PC}$ has been implicated in a number of specific cell biological processes. PC plays an important role in signal transduction as a source of lipid signaling molecules, such as lysoPC, phosphatidic acid (PA), and diacylglycerol (DAG) [3,4]. In yeast defects in the biosynthesis of PC lead to respiratory deficient cells that form tiny colonies in glucose medium, socalled petites [5]. Recently, PC was suggested to be involved in a specific lipid-protein interaction with the yeast mitochondrial glycerol-3-phosphate dehydrogenase Gut2 based on the results of a photolabeling approach [6].

E-mail address: a.i.p.m.dekroon@chem.uu.nl.
The glycerophospholipid composition of yeast membranes is similar to that of higher eukaryotes with PC, phosphatidylethanolamine (PE), phosphatidylinositol (PI), phosphatidylserine (PS), and cardiolipin representing the major classes. The glycerophospholipid biosynthetic pathways in yeast are well established and for the most part similar to those in higher eukaryotes [7]. Combined with the ease of genetic manipulation, this makes yeast the model eukaryote of choice for studying PC metabolism at the level of the molecular species.

A phospholipid class such as PC is not a single entity: it is composed of a number of molecular species that vary by the combinations of acyl chains that are esterified at the $s n-1$ and $s n-2$ position of the glycerol backbone. The repertoire of fatty acids in Saccharomyces cerevisiae is simple compared to that in higher eukaryotes and results from synthesis, elongation, and Ole1p-mediated desaturation. In yeast, phospholipids primarily contain C16:0, C16:1, C18:0 and C18:1 fatty acids with minor contributions of $\mathrm{C} 14: 0$, and $\mathrm{C} 14: 1$. The saturated acyl chains are mostly attached at the $s n-1$ position, while unsaturated fatty acids predominate at the $s n-2$ position [8,9].

The phospholipid class composition and the composition of the lipid acyl chains determine to a large extent the physical properties of biological membranes. Compositions differ 
between different membranes and membrane leaflets, and are subject to adaptation in response to changing environments, particularly in unicellular organisms without means of active mobility such as yeast. The physical properties of membranes include membrane thickness, intrinsic curvature, and fluidity, which affect membrane barrier function, the activity of membrane associated enzymes, and dynamic processes such as membrane fusion and fission.

In view of the sheer abundance of PC in wild type yeast, the PC species profile, i.e. the ensemble of PC molecules with different acyl chain compositions, is a major determinant of the physical properties of the membrane. The advent of electrospray ionisation tandem mass spectrometry has greatly facilitated the analysis of lipid classes at the species level [10]. This review will focus on the contributions of PC biosynthesis and turnover to establishing $\mathrm{PC}$ homeostasis at the level of the molecular species in the model eukaryote yeast. Advantages of using baker's yeast in the analysis of lipids at the level of molecular species by mass spectrometry include the relatively simple repertoire of acyl chains, the lack of sphingomyelin and of alkyl-linked glycerophospholipids, the efficient incorporation of (labeled) lipid precursors, and the absence of other metabolic roles of choline than that of precursor and degradation product of PC.

Saccharomyces cerevisiae is extremely tolerant with respect to its membrane lipid composition. Acyl chain compositions of yeast cells differ significantly between different wild type strains [11], and vary with the culture medium $[9,12]$, and the carbon source used to culture the cells [13]. Moreover, the lipid composition is easily manipulated by culturing yeast in the presence of specific fatty acid supplements [14,15]. The fact that of the major phospholipid classes only PI [16], PE [17,18], and some form of methylated PE (i.e. PC, its monomethylated precursor PMME, or its dimethylated precursor PDME) $[19,20]$ are essential, underscores the versatility of yeast in adapting its membrane lipid composition. The final part of this review will address the role of PC in the interplay between phospholipid class and acyl chain composition in yeast membranes. New insights obtained in a PC biosynthesis mutant point to a regulatory mechanism for balancing the membranes' contents of bilayer and non-bilayer lipids in order to maintain membrane intrinsic curvature in an optimal range.

\section{Biosynthesis of PC}

\subsection{Two biosynthetic pathways}

The biosynthesis of $\mathrm{PC}$ in yeast proceeds via two pathways (reviewed in [7]). PC is the end product of the CDP-DAG route for phospholipid synthesis (Fig. 1), in which CDP-DAG is first converted to PS by PS synthase (Cholp/Pss1p) in the ER and in one of its subdomains, the mitochondria associated membrane (MAM) [21]. Subsequently, PS is decarboxylated to PE by a PS decarboxylase, either by Psd1p localized in the mitochondrial inner membrane [22] or by Psd $2 p$ localized in the Golgi/vacuole [23]. Finally, PE is methylated to PC in three steps by two methyltransferases localized to the ER that use $S$ adenosyl methionine (SAM) as methyl donor. The first

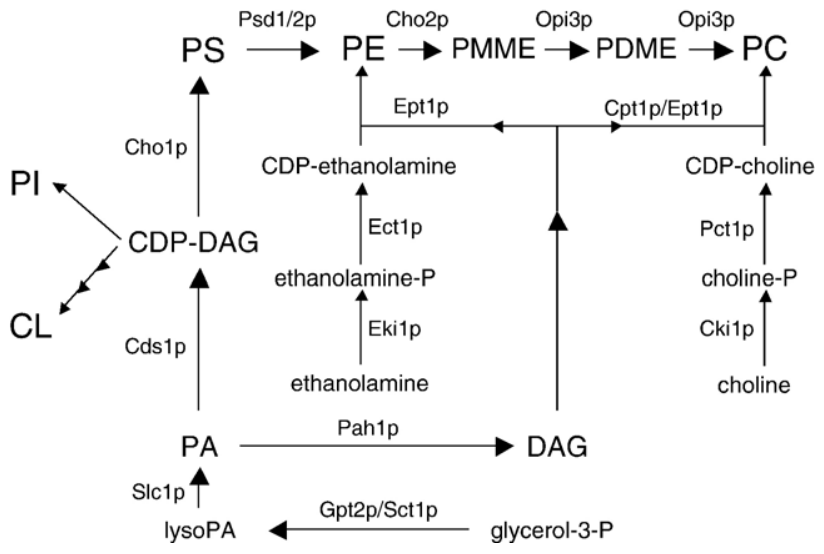

Fig. 1. Biosynthetic pathways for the major membrane phospholipids (bold) in yeast. Enzymes characterized at the gene level contributing to the synthesis of $\mathrm{PC}$ are indicated.

methylation is catalyzed by PE methyltransferase (Pemlp/ Cho2p) yielding phosphatidylmonomethylethanolamine (PMME), and the second and third by phospholipid methyltransferase (Pem2p/Opi3p) yielding phosphatidyldimethylethanolamine (PDME) and PC, respectively $[19,24,25]$.

In the second pathway, the CDP-choline branch of the Kennedy pathway, PC is produced from diacylglycerol and choline (Fig. 1). Choline is first phosphorylated by the cytosolic kinase Ckilp. Next, phosphocholine is converted to CDPcholine by Pctlp, a membrane-associated enzyme localized to the nuclear periphery [26-28], in the rate-limiting step of the CDP-choline pathway under conditions of choline limitation [27]. The cholinephosphotransferase Cptlp of which the subcellular localization has not been unequivocally determined yet $[28,29]$, catalyzes the final step in which the phosphocholine moiety is transferred to diacylglycerol yielding PC. Diacylglycerol derives from the dephosphorylation of PA [30,31], from turnover of phosphoinositides by phospholipase $\mathrm{C}$ [32], from deacylation of triacylglycerol [33], and from the transfer of the inositol phosphate head group from PI to ceramide yielding inositol phosphorylceramide in sphingolipid biosynthesis (reviewed in [34]). Ckilp and Cpt1p share overlapping substrate specificities with their counterparts from the CDPethanolamine pathway, Ekilp and Ept1p, respectively, that may also contribute to PC synthesis [35,36]. These pairs of enzymes most likely evolved from common ancestors by whole genome duplication [37]. In contrast, Pctlp and the cytidylyltransferase from the CDP-ethanolamine pathway Ect1p do not show crosspathway activity $[38,39]$.

The net contribution of the CDP-choline route to cellular PC depends on, and increases with the availability of choline in the culture medium [27]. Exogenous choline is taken up by active transport via the high affinity transporter Hnmlp [40]. In the absence of exogenous choline the CDP-choline route is active in recycling choline from PC turnover, as was deduced from the increased incorporation of radiolabeled phosphate in $\mathrm{PC}$ in wild type as compared to cptl cells cultured in the absence of choline, while the incorporation of radiolabel from serine was unaffected [41]. 
Radiolabeling of yeast cells with phospholipid precursors followed by subcellular fractionation has been used to assess the contributions of the PC biosynthetic pathways to the PC pools in subcellular compartments. These studies revealed that the proportions of $\mathrm{PC}$ produced by the two routes are similar in total cellular membranes, microsomes, and mitochondrial membranes, suggesting that $\mathrm{PC}$ is evenly distributed from a single pool $[42,43]$. Consistent with this notion, the molecular species profiles of PC in different subcellular membranes are similar $[44,45]$.

The two PC biosynthetic pathways in yeast can substitute for one another. Mutants in which the methylation of PE is impaired have wild type PC levels and grow at almost wild type rates provided that choline is supplied in the medium $[19,46]$. Mutants lacking a functional CDP-choline pathway increase the rate of phospholipid synthesis via the CDP-DAG route, and exhibit only a slightly slower rate of growth $[5,47]$. What would be the advantage for yeast to maintain two PC biosynthetic pathways? So far, few functional differences between the two PC biosynthetic routes have been reported. The long held view that inactivation of the CDP-choline route but not of the methylation pathway suppresses the temperature-sensitive secretion defect of $\sec 14^{\text {ts }}$ mutants, was refuted [48].

From the perspective of PC as bulk membrane lipid, the availability of two biosynthetic routes could offer versatility in regulating the molecular species composition of $\mathrm{PC}$, provided that the methylation of PE and the CDP-choline yield different products in terms of PC molecular species. Recent findings from this laboratory summarized below indicate that this is indeed the case.

\subsection{PC biosynthesis at the molecular species level}

The technique of electrospray ionisation tandem mass spectrometry (ESI-MS/MS) has enabled quantitative analysis at the molecular species level of individual phospholipid classes in total lipid extracts, by taking advantage of class-specific fragmentations upon collisionally activated dissociation (CID) [10]. By applying this technique to yeast lipid extracts, it was demonstrated that phospholipid classes that are metabolically closely related have significant differences in acyl chain profiles, indicating that the molecular species profiles of phospholipid classes are tightly regulated [44].

The molecular species of PC are detected by parent ion scanning for $m / z 184$ in the positive ion mode [10]. In this mode, all molecular ions that upon CID release a fragment of $\mathrm{m} / \mathrm{z} 184$ corresponding to the protonated phosphocholine headgroup, are recorded. The PC species profile in yeast contains four prominent components (Fig. 2B, black bars): di-unsaturated 32:2 (containing two C16:1 acyl chains) and 34:2 (containing a

A

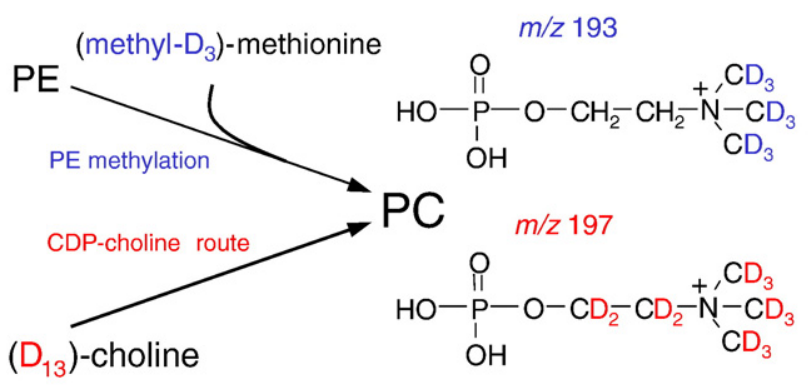

B

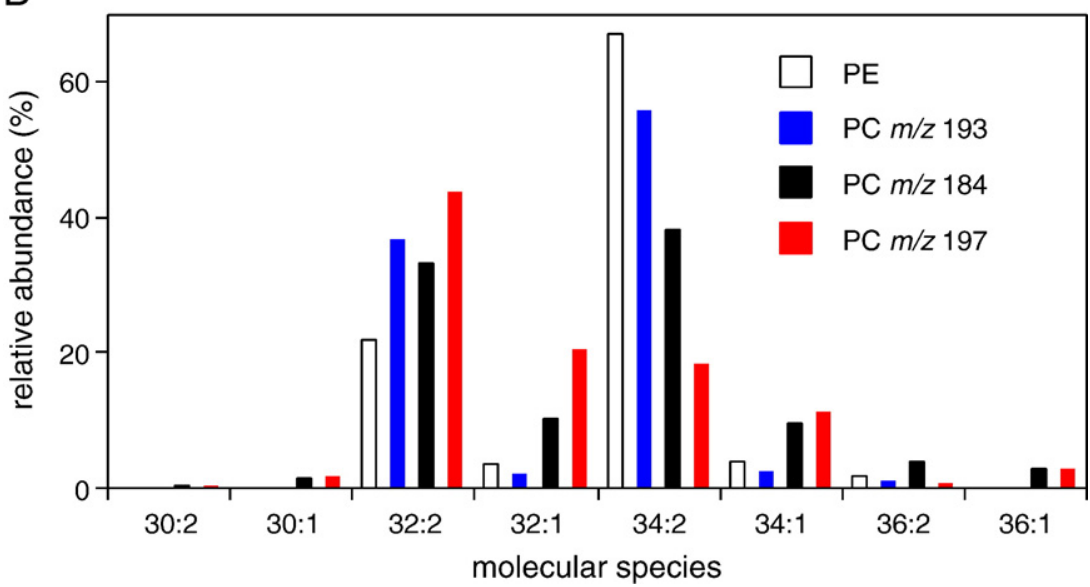

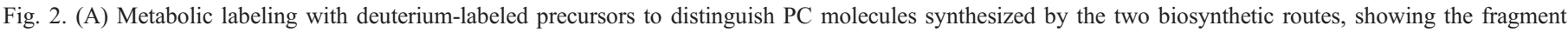

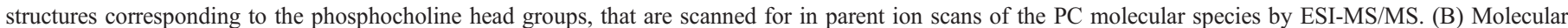

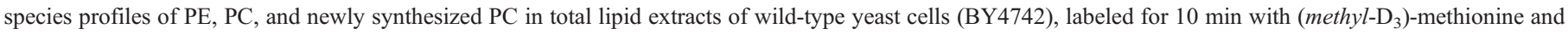

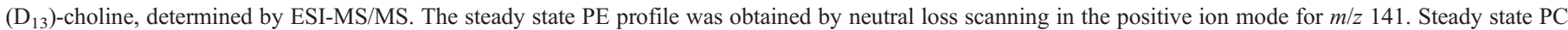

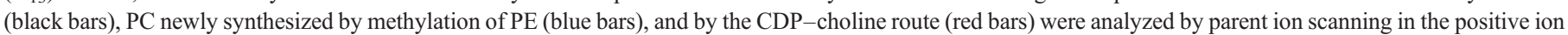
mode for $m / z 184,193$, and 197, respectively. Data were taken from Ref. [49]. 
C16:1 and a C18:1 acyl chain), and the mono-unsaturated species 32:1 (composed of $\mathrm{C} 16: 0$ and $\mathrm{C} 16: 1$ ) and $34: 1$ (composed of C16:0 and C18:1 and/or of C18:0 and C16:1) [49]. Note that with the exception of 32:2, these components may correspond to two (32:1 and 34:2) or four (34:1) molecular species due to positional isomers.

Pulse labeling of cells with stable isotope-labeled lipid precursors and subsequent analysis by ESI-MS/MS allows distinction between newly synthesized and pre-existing pools of most phospholipid classes, based on the difference in molecular mass conferred by the isotope labels [49,50]. The contributions of the methylation of $\mathrm{PE}$ and the CDPcholine pathway to the PC species profile were distinguished by pulsing wild type yeast for $10 \mathrm{~min}$ with deuterium-labeled (methyl- $\left.\mathrm{D}_{3}\right)$-methionine and $\left(\mathrm{D}_{13}\right)$-choline, respectively (Fig. 2A). Whereas PE methylation predominantly yielded di-unsaturated $\mathrm{PC}$, the $\mathrm{CDP}$-choline route produced a more diverse profile of PC species (Fig. 2B). The steady state distribution of PC molecular species in wild type cells could not be fitted to any weighted average of the species profiles contributed by the two biosynthetic pathways (Fig. 2B), implicating other metabolic processes such as species-selective turnover and/or acyl chain remodeling of PC [49].

\subsection{Species-selectivity of the PC biosynthetic enzymes}

ESI-MS/MS combined with stable isotope labeling has enabled detection at the species level of the substrates used by phospholipid biosynthetic enzymes in vivo. To investigate the influence of the methyltransferases on the species profile of the PC they produce, the species profile of PC newly synthesized by the methylation pathway was compared to that of its precursor PE that was determined by neutral loss scanning in the positive ion mode for $m / z$ 141. In this scanning mode, all molecular ions that upon CID loose a neutral fragment of $\mathrm{m} / \mathrm{z}$ 141 corresponding to the phosphoethanolamine headgroup are recorded [10]. Compared to the species profile of cellular (or microsomal) PE that is dominated by 32:2 PE $(\sim 20 \%)$ and $34: 2$ PE $(\sim 65 \%)$, the derived newly synthesized PC is enriched in the $32: 2$ species $(\sim 35 \%$, Fig. $2 \mathrm{~B})$, suggesting that the methyltransferases preferentially convert 32:2 PE. When the species composition of the PE precursor pool was varied, by using different carbon sources or a phospholipid biosynthetic mutant strain, similar enrichments were observed in newly synthesized PC [51].

A similar preferential conversion of 32:2 $\mathrm{PE}$ to $\mathrm{PC}$ was detected in vitro, upon labeling isolated microsomes with $S$ (methyl- $\mathrm{D}_{3}$ )-adenosyl-methionine, indicating that it is an intrinsic property of the methyltransferases in microsomes, and that it is not due to species-selectivity in the intermembrane transport processes required to supply the methyltransferases with PE [51]. PE is for the most part synthesized by mitochondrial Psd1p and by Psd2p in the Golgi/vacuole $[17,23]$, and its conversion to PC requires intermembrane transport to the ER (reviewed in [52]. Consistent with the above notion, a recent analysis of the species distributions of the aminoglycerophospholipids in yeast subcellular fractions did not reveal species-selectivity in interorganelle phospholipid transport [45]. The preferential methylation of 32:2 PE is for the most part conferred by Cho2 $p$ with a minor contribution by Opi3p [51].

Interestingly, it was recently reported that deletion of the PSD2 gene reduced the contribution of PE methylation to cellular PC production to a much larger extent than deletion of PSD1 [42], while Psd1p is generally considered the main source of PE synthesis in yeast $[17,53]$. Pulse labeling with stable isotope labeled L-serine of $p s d 1$ and $p s d 2$ mutants and analysis of the labeled PE species synthesized by ESI-MS/MS could shed a light on the possibility that differences in species composition between the PE pools produced by Psdlp and Psd2p account for the preferred methylation of PE derived from Psd2p. The similarity in PE species profiles between $p s d 1$ and $p s d 2$ strains [45] seems to argue against this possibility.

In contrast to the methyltransferases [54], the aminoalcohol phosphotransferases catalyzing $\mathrm{PC}$ synthesis in the CDPcholine route, Cpt1p and Eptlp, retain enzyme activity in the presence of detergents. The substrate specificities of Cptlp and Eptlp were assayed in vitro, in a mixed micellar system containing chemically defined DAG species [55]. The conversion rate of Cptlp was highest for the shorter species (32:2>32:0 34:1>36:2), whereas Ept1p showed the highest activity toward di-unsaturated species $(36: 2>32: 2>34: 1)$.

Due to the small size and rapid turnover of the cellular DAG pool, it is not possible to examine the precursorproduct relationships at the species level in the CDPcholine pathway in intact cells. ESI-MS/MS analysis of PC newly synthesized by ept 1 and cpt 1 cells during a pulse with $\left(\mathrm{D}_{13}\right)$-choline revealed that $\mathrm{Cpt} 1 \mathrm{p}$ and Eptlp produce the four major PC species in different ratios with 32:2 PC and 34:2 PC as most abundant species, respectively [56]. The differences in the species profiles were consistent with the above in vitro results [55], arguing that they result from differences in substrate specificity. Yet, it cannot be excluded that Eptlp and Cptlp consume distinct sets of DAG species due to different subcellular localizations giving access to separate pools of DAG differing in species composition.

While Cpt1p does not have significant ethanolamine phosphotransferase activity, Eptlp can transfer both phosphoethanolamine and phosphocholine to DAG [36]. The nature of the CDP-aminoalcohol donor does not influence the selectivity in DAG substrate use by Ept1 neither in vitro [55], nor in vivo [56]. The relative contributions of Ept1p and Cpt1p to $\mathrm{PC}$ synthesis via the $\mathrm{CDP}$-choline route will affect PC species homeostasis. The species profile of newly synthesized PC in the eptl strain was almost identical to that in the parental wild type, and differed significantly from that obtained in the cpt1 strain in which 34:2 PC was the major species [56], indicating that Eptlp does not contribute substantially to PC biosynthesis in the BY4742 strain background. The contribution of Ept1p to net PC synthesis was previously found to depend on the strain background, varying from $5 \%$ or less [41] to as much as $60 \%$ [57]. 


\section{Metabolic fates of PC}

The steady state distribution of PC molecular species in wild type cells cannot be accounted for by contributions of the two biosynthetic pathways only (Section 2.2; [49]. The steady state PC species profiles of yeast strains lacking either of the two PC biosynthesis routes (pct1 and cho2opi3) are very similar to wild type even though the methylation of PE and the CDP-choline route produce the PC species in different ratios $[49,56]$. Therefore additional processes have to be involved in maintaining PC homeostasis at the species level. The acyl chain composition of the (newly synthesized) PC pool could be remodeled by species-selective degradation, and/or by exchange of acyl chains. Sections 3.1 and 3.2 provide an overview of the metabolic conversions of $\mathrm{PC}$, and comment on their potential influence on the PC species profile.

\subsection{PC degradation}

Knowledge on the metabolic processes downstream of PC synthesis in yeast is fairly limited. The turnover of PC by the SPO14-encoded phospholipase D yields phosphatidic acid and choline, and is essential for sporulation [58,59]. Moreover, Spo14p activity is essential for yeast suppressor mutants that bypass the essential requirement for Sec14p in secretion $[60,61]$. The only other phospholipase D activity in yeast that so far escaped identification at the gene level preferentially cleaves PE and PS $[62,63]$. PC also serves as substrate for phospholipases B yielding glycerophosphocholine (GPC) and two fatty acids (see also review by Patton-Vogt in this issue). Yeast contains at least 4 genes encoding phospholipases B, neither of which is essential. Plblp is localized at the plasma membrane and in the periplasmic space, and was shown to deacylate PC in vivo with the GPC produced released into the culture medium [64]. Plb2p and Plb3p are homologous to Plb1p and also localize to the cell surface. Plb2p was shown to degrade PC only in vitro $[65,66]$, while Plb3p does not convert $\mathrm{PC}$ at a detectable rate [66].

The phospholipase B Nte1p, a homologue of human neuropathy target esterase localized to the ER, is responsible for intracellular GPC production [67]. Its activity is induced by the presence of choline in the culture medium and by raising the growth temperature to $37^{\circ} \mathrm{C}$, conditions that enhance PC synthesis by the $\mathrm{CDP}$-choline route and not the methylation pathway [68]. Intriguingly, Nte1p selectively degrades PC produced by the $\mathrm{CDP}$-choline pathway, not by the methylation of PE $[67,68]$. The cause of this selectivity is presently not understood. Interestingly, deletion of the GPT2 (GAT1) and SCT1 (GAT2) genes encoding the major glycerol-3-phosphate acyltransferases (Fig. 1), enhance and decrease the deacylation of CDP-choline-derived PC to GPC, respectively [69]. Recently, the GDE1 gene was identified that encodes the phosphodiesterase responsible for metabolizing intracellular GPC to choline [70,71]. Whether reacylation of GPC occurs is not known.

When choline is absent from the culture medium, turnover of PE-derived PC by phospholipases D and/or B, yields choline for use in the CDP-choline pathway [41], thus contributing to the species composition of PC (Fig. 3). Whether Spo14p, Plblp, and Nte1p are species-selective and thereby directly affect the PC species profile has not been documented. However, it is tempting to speculate that the exclusive degradation of CDPcholine derived PC by Ntelp is based on species selectivity. During prolonged labeling with deuterated PC precursors, no change in species profile of the pre-existing PC pool was observed in wild type cells cultured at $30^{\circ} \mathrm{C}$, arguing against the occurrence of extensive species selective degradation [49].

\section{2. $P C$ remodeling by acyl chain exchange}

Remodeling by acyl chain exchange is generally thought to proceed via deacylation to a lyso-phospholipid intermediate by a phospholipase A (or B), followed by an acyl-CoA dependent reacylation by an acyltransferase. Alternatively, acyl chain shuffling via transacylation may occur in which a phospholipid serves as acyl chain donor and a lysophospholipid as the acceptor [72].

Reports on phospholipases A in yeast are scarce. Mitochondria-associated $\mathrm{Ca}^{2+}$-independent phospholipases $\mathrm{A} 1$ and $\mathrm{A} 2$ hydrolyzing PC were reported $[73,74]$, however, so far no gene-enzyme relationships were established. Another reaction yielding lyso-PC is the transfer of an acyl chain from PC to DAG to synthesize triglycerides, which is catalyzed by the phospholipid-diacylglycerol acyltransferase Lrolp in vitro $[75,76]$.

Yamada and coauthors [8] were the first to report acyl-CoA dependent $s n$-1-acyl-glycero-phosphocholine ( $s n$-1-acyl-GPC) and $s n$-2-acyl-GPC acyltransferase activities in Saccharomyces cerevisiae that were localized to microsomes. Wagner and Paltauf [9] provided evidence for the occurrence of phospholipase A-mediated deacylation followed by reacylation in vivo, by incubating yeast cells with radiolabeled palmitic and oleic acid. Already after $2 \mathrm{~min}$, incorporation of both acyl chains was detected in the major phospholipid classes, the extent of labeling being highest in PI and PC. Subsequent esterification

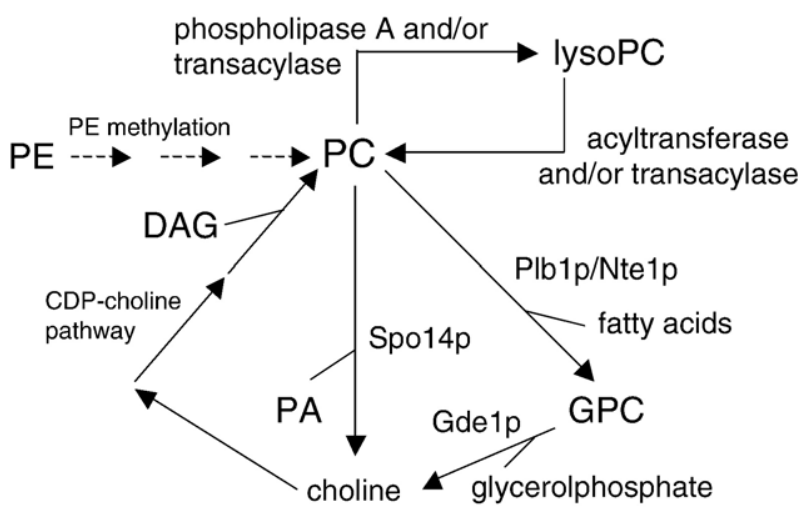

Fig. 3. Metabolic fates of PC in vivo. PC can be degraded by a phospholipase D (Spo14p) or by phospholipases B (Plb1p, Ntelp) and a phosphodiesterase (Gdelp) to yield choline that can be reincorporated into PC via the CDP-choline pathway. Remodeling of PC by acyl chain exchange most likely proceeds via deacylation to lysoPC followed by reacylation as indicated. The enzymes catalyzing acyl chain exchange remain to be identified. 
and transesterification of the free fatty acid and the lysoPC, respectively, obtained after treating the $\mathrm{PC}$ fraction with phospholipase A2, revealed that oleic acid was almost exclusively incorporated at the $s n-2$ position of PC (99\%). The preferential incorporation at the PC $s n-2$ position of the label from palmitic acid that for the most part had been converted to palmitoleic acid, was slightly less $(90 \%)$. Although this study does not provide quantitative information as to the relative importance of acyl chain exchange in establishing the PC species profile, it was the first to show selective acyl chain exchange.

Richard and McMaster [77] characterized a microsomal, acyl-CoA dependent lysoPC acyltransferase activity utilizing a broad range of $s n$-1-lysoPC acyl chain lengths. In addition, they demonstrated lysoPC acyltransferase activity in vivo by the conversion of radiolabeled $s n-1-O$-alkyl-GPC to PC in a $p l b 1$ strain. Apart from exhibiting phospholipase B activity, Plb1p was shown to have acyl-CoA independent transacylase activity as demonstrated by its ability to convert $s n$-1-lyso-PC into PC in vitro [64]. Recently, the TAZ1 gene product, a predicted acyltransferase localized to the mitochondrial outer membrane [78] and implicated in acyl chain remodeling of cardiolipin [79], was shown to exhibit acyl-CoA independent $s n$-1-lysoPC acyltransferase activity upon expression in E. coli [80]. The enzyme activity was found associated with the mitochondrial fraction from wild type cells but not from a tazl deletion mutant.

To study PC remodeling in vivo, we used a pctl strain, lacking a functional $\mathrm{CDP}$-choline route to preclude recycling into $\mathrm{PC}$ of choline originating from degradation of PC synthesized by the methylation of PE. Labeling pct1 cells for 10 min with (methyl- $\left.\mathrm{D}_{3}\right)$-methionine followed by ESI-MS/MS analysis of the newly synthesized PC, revealed a species profile dominated by $32: 2$ and 34:2 PC and containing relatively minor proportions of 32:1 and 34:1 PC. During continued labeling, the species profile of deuterated PC gradually evolved to a profile resembling that of steady state $\mathrm{PC}$ with at least twice the initial amounts of 32:1 and 34:1 [49]. Species selective degradation alone is unlikely to account for this process, as it would involve degradation of some $50 \%$ of newly synthesized PC. This leaves remodeling by acyl chain exchange as the more plausible mechanism. ESI-MS/MS analysis of lysoPC after treatment of the pct1 lipid extracts with phospholipase A2, revealed the substitution of C16:1 acyl chains esterified at the $s n-1$ position of newly synthesized PC by other acyl chains, unequivocally demonstrating the contribution of acyl chain remodeling by acyl chain exchange to the PC species profile [49]. In Fig. 3 the metabolic conversions of PC have been summarized.

\section{The role of $P C$ in balancing membrane lipid composition}

From the perspective of overall membrane lipid homeostasis, both PC biosynthetic routes are complementary in that they affect the balance between bilayer and non-bilayer favouring lipids. The lipid precursors of the bilayer preferring lipid $\mathrm{PC}$ in the methylation and the CDP-choline pathway, PE and DAG, respectively, are both type II lipids that have a conical molecular shape resulting from the comparatively small cross-sectional area of the polar headgroup, and consequently confer negative curvature stress to biological membranes [81]. Isolated type II lipids organize as reversed non-lamellar structures such as the hexagonal $\mathrm{H}_{\text {II }}$ phase when hydrated [2]. Membrane intrinsic curvature is important for membrane processes such as fusion and fission, and for the proper assembly and function of membrane proteins (reviewed in [82]). In yeast, the importance of type II lipids in membrane function is supported by the finding that a reduced PE content in mutants defective in PE synthesis, is deleterious for growth $[17,18]$. Moreover, it was recently found that deletion of the two genes encoding mitochondrial enzymes producing non-bilayer phospholipids, CRD1 and PSD1, is synthetically lethal [83]. On the other hand, an (local) excess of type II lipids could interfere with the barrier function of the membrane. In contrast to PE, DAG is only a minor membrane lipid. However, as DAG has been implicated in protein secretion from the Golgi complex [84] and in vacuole fusion [85], regulation of local DAG levels is required.

To understand the role of PC in membrane lipid homeostasis, the response to a gradual lowering of the PC content was investigated by depriving the choline auxotroph cho2opi3 mutant of choline [20]. Cell growth continued at a normal rate for 4-5 generations until the PC level had dropped below $2 \%$ of total phospholipids. In this time window a remarkable shift in cellular acyl chain composition occurred with the acyl chains becoming shorter and more saturated. The changes were largest in PE, the most abundant phospholipid under conditions of PC depletion (Fig. 4A). The bilayer to hexagonal $\mathrm{H}_{\text {II }}$ phase transition temperature of isolated $\mathrm{PE}$ fractions increased from about $20{ }^{\circ} \mathrm{C}$ to $50{ }^{\circ} \mathrm{C}$ as the PE level rose from $20 \%$ to $50 \%$ of total phospholipids [20], in agreement with shortening and increased saturation of the acyl chains decreasing the nonbilayer propensity of PE [86].

The concomitant shortening and increased saturation of the acyl chains do not reflect a unidirectional change in membrane fluidity. On the contrary, to maintain membrane fluidity in response to decreasing temperatures, shortening and increased desaturation of the acyl chains were reported [87,88]. The isothermal changes in acyl chain composition in response to PC depletion are consistent with the need to keep intrinsic membrane curvature in an optimal range by maintaining the right balance between bilayer and non-bilayer preferring phospholipids (Fig. 4B) [20], an established regulatory mechanism in prokaryotes [89].

Changes in $\mathrm{PE} / \mathrm{PC}$ ratios reported in yeast cultures supplemented with specific fatty acids support the proposed regulatory mechanism for membrane intrinsic curvature. Growth on myristoleic acid (C14:1) induced an enormous increase in the $\mathrm{PE} / \mathrm{PC}$ ratio of wild type yeast as compared to growth on C16:1 (4.1 vs. 0.8) [90], counterbalancing the decrease in non-bilayer propensity of $\mathrm{PE}$ resulting from shorter acyl chains. Similarly, the PE/PC ratio of a fatty acid auxotroph was higher when supplemented with trans C16:1 than with cis 16:1 (1.1 vs. 0.6) [15] to compensate for the loss of non-bilayer 
A

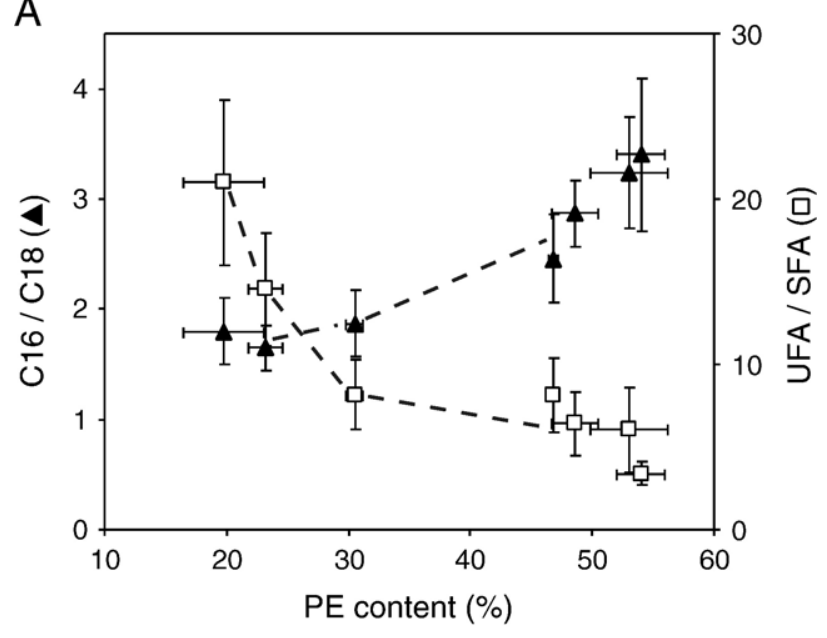

B
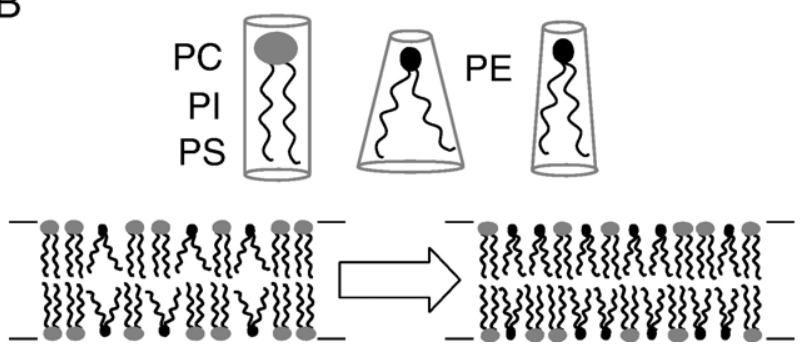

Fig. 4. (A) The increase in PE content induced by PC depletion in cho2opi3 cells is accompanied by shortening and increased saturation of the PE acyl chains. The ratios of $\mathrm{C} 16$ to $\mathrm{C} 18$, and of unsaturated to saturated (UFA/SFA) acyl chains were calculated from ESI-MS/MS data, and plotted against the PE content expressed as percentage of total phospholipids. Data points are connected with dashed lines to guide the eye. Data were taken from ref. [20]. (B) Schematic representation of the molecular shapes of the major membrane phospholipids and of the changes in membrane lipid composition occurring during PC depletion in cho2opi3 cells. As the content of the bilayer lipid PC drops and the level of the non-bilayer lipid PE increases, PE looses non-bilayer propensity, depicted here by a reduction in cone shape, in order to keep membrane intrinsic curvature balanced. See text for further comments.

propensity of PE with trans unsaturated fatty acyl chains. Conversely, the PE content tends to decrease when yeast is supplemented with unsaturated fatty acids of increasing length and degree of unsaturation [14], which enhance the non-bilayer propensity of PE.

A mutation in the RSP5 gene encoding a ubiquitin ligase involved in the regulation of fatty acid desaturation by Ole1p, leads to accumulation of saturated fatty acids, and is suppressed by overexpression of the PIS1 gene encoding PI synthase [91]. The suppression was explained by a restoration of the unsaturation/saturation ratio of $\mathrm{PE}$ that results from rerouting the saturated fatty acids into PI, and is most likely another manifestation of the requirement to safeguard PE from loosing non-bilayer propensity and balance membrane intrinsic curvature.

PC is not essential in yeast, however, some form of methylated PE appears to be required for growth. PC deficiency can be bypassed by its biosynthetic precursor in the methylation pathway PDME provided that the yeast cells are grown in the presence of inositol, substrate for the synthesis of PI $[19,20]$. Like PC, PI is a bilayer forming phospholipid [92] that compensates for the replacement of PC by PDME that forms somewhat less stable bilayers [93]. The ability of PMME to replace $\mathrm{PC}$ in the presence of inositol appears to depend on the strain background $[19,20]$. A twist to the story came with the recent report that phosphatidylpropanolamine, a nonnatural, non-bilayer forming phospholipids, could replace the methylated phospholipids altogether in a cho2opi3 strain [94]. Cells cultured in the presence of propanolamine exhibit a strong increase in the level of the bilayer lipid PI, and a shift from C34 to $\mathrm{C} 32$ species in the species profile of PE, in keeping with a regulatory mechanism balancing the proportions of bilayer and non-bilayer lipids.

The effects exerted by phospholipid class composition on molecular species composition and vice versa described above, highlight the role of PC as bilayer stabilizing membrane constituent counterbalancing type II membrane lipids to maintain membrane intrinsic curvature in an optimal window. From the point of view of regulating membrane intrinsic curvature, the preferential methylation of 32:2 PE over 34:2 PE to PC described in Section 2.3 [51] could be regarded as enhancing the non-bilayer propensity of PE. Based on this notion it is tempting to speculate that the phospholipid methyltransferases Cho2p and Opi3p may represent first line regulators of membrane intrinsic curvature.

\section{Conclusion and future directions}

Biosynthesis, turnover, and acyl chain remodeling of phospholipids are important determinants of membrane lipid composition. With the recent advances in the analysis of lipids by mass spectrometry it is now possible to study lipid metabolism at the level of the molecular species. Combined with stable isotope labeling, this allows us to determine the contributions of the various biosynthetic routes in terms of phospholipid species, to visualize the substrate selectivity of individual phospholipid biosynthetic enzymes in vivo, and to monitor remodeling of acyl chains, as exemplified for PC in this review. This powerful technology will be an essential tool in uncovering the identity and function of the remodeling enzymes that adjust the species composition of PC and other lipids.

$\mathrm{PC}$ is a key player in balancing the proportions of bilayer and non-bilayer lipids that determine membrane intrinsic curvature. The balance between bilayer and non-bilayer lipids has been recognized as a novel criterion in the regulation of yeast membrane lipid composition. To keep physical membrane properties such as membrane intrinsic curvature in the optimal range, yeast must possess an intricate regulatory network for reciprocally tuning phospholipid class and acyl chain composition. One of the challenges facing us is to resolve this regulatory network and the underlying membrane sensors, and to figure out how it interfaces with the known regulatory mechanisms of phospholipid and fatty acid biosynthesis [95] [Martin et al. in this issue; Tehlivets et al. in this issue]. With its unsurpassed ability to tolerate a wide range of different lipid compositions, 
yeast continues to be a valuable model eukaryote for investigating membrane lipid homeostasis.

\section{Acknowledgments}

I thank the former and current members of the yeast lipid group in the Dept. Biochemistry of Membranes, Marjolein Janssen, Henry Boumann, Martijn Koorengevel, Pieter Rijken, Jacob Gubbens, and Cedric De Smet, for their valuable contributions, and Ben de Kruijff for continued interest and support. This work was supported by the Division of Chemical Sciences and by the Division of Earth and Life Sciences in the Netherlands, with financial aid from the Netherlands Organization for Scientific Research.

\section{References}

[1] C. Kent, G.M. Carman, Interactions among pathways for phosphatidylcholine metabolism, CTP synthesis and secretion through the Golgi apparatus, Trends Biochem. Sci. 24 (1999) 146-150.

[2] P.R. Cullis, B. de Kruijff, Lipid polymorphism and the functional roles of lipids in biological membranes, Biochim. Biophys. Acta 559 (1979) 399-420.

[3] J.H. Exton, Phosphatidylcholine breakdown and signal transduction, Biochim. Biophys. Acta 1212 (1994) 26-42.

[4] Z. Cui, M. Houweling, Phosphatidylcholine and cell death, Biochim. Biophys. Acta 1585 (2002) 87-96.

[5] P. Griac, M.J. Swede, S.A. Henry, The role of phosphatidylcholine biosynthesis in the regulation of the INO1 gene of yeast, J. Biol. Chem. 271 (1996) 25692-25698.

[6] M.J. Janssen, F. van Voorst, G.E. Ploeger, P.M. Larsen, M.R. Larsen, A.I. de Kroon, B. de Kruijff, Photolabeling identifies an interaction between phosphatidylcholine and glycerol-3-phosphate dehydrogenase (Gut2p) in yeast mitochondria, Biochemistry 41 (2002) 5702-5711.

[7] G.M. Carman, S.A. Henry, Phospholipid biosynthesis in the yeast Saccharomyces cerevisiae and interrelationship with other metabolic processes, Prog. Lipid Res. 38 (1999) 361-399.

[8] K. Yamada, H. Okuyama, Y. Endo, H. Ikezawa, Acyltransferase systems involved in phospholipid metabolism in Saccharomyces cerevisiae, Arch. Biochem. Biophys. 183 (1977) 281-289.

[9] S. Wagner, F. Paltauf, Generation of glycerophospholipid molecular species in the yeast Saccharomyces cerevisiae. Fatty acid pattern of phospholipid classes and selective acyl turnover at $s n-1$ and $s n-2$ positions, Yeast 10 (1994) 1429-1437.

[10] B. Brügger, G. Erben, R. Sandhoff, F.T. Wieland, W.D. Lehmann, Quantitative analysis of biological membrane lipids at the low picomole level by nano-electrospray ionization tandem mass spectrometry, Proc. Natl. Acad. Sci. U. S. A. 94 (1997) 2339-2344.

[11] G. Daum, G. Tuller, T. Nemec, C. Hrastnik, G. Balliano, L. Cattel, P. Milla, F. Rocco, A. Conzelmann, C. Vionnet, D.E. Kelly, S. Kelly, E. Schweizer, H.J. Schuller, U. Hojad, E. Greiner, K. Finger, Systematic analysis of yeast strains with possible defects in lipid metabolism, Yeast 15 (1999) 601-614.

[12] M.T. Chatterjee, S.A. Khalawan, B.P. Curran, Subtle alterations in growth medium composition can dramatically alter the percentage of unsaturated fatty acids in the yeast Saccharomyces cerevisiae, Yeast 18 (2001) 81-88.

[13] G. Tuller, T. Nemec, C. Hrastnik, G. Daum, Lipid composition of subcellular membranes of an FY1679-derived haploid yeast wild-type strain grown on different carbon sources, Yeast 15 (1999) 1555-1564.

[14] B.J. Pilkington, A.H. Rose, Incorporation of unsaturated fatty acids by Saccharomyces cerevisiae: conservation of fatty-acyl saturation in phosphatidylinositol, Yeast 7 (1991) 489-494.

[15] B.S. Tung, E.R. Unger, B. Levin, T.A. Brasitus, G.S. Getz, Use of an unsaturated fatty acid auxotroph of Saccharomyces cerevisiae to modify the lipid composition and function of mitochondrial membranes, J. Lipid Res. 32 (1991) 1025-1038.

[16] J. Nikawa, T. Kodaki, S. Yamashita, Primary structure and disruption of the phosphatidylinositol synthase gene of Saccharomyces cerevisiae, J. Biol. Chem. 262 (1987) 4876-4881.

[17] R. Birner, M. Burgermeister, R. Schneiter, G. Daum, Roles of phosphatidylethanolamine and of its several biosynthetic pathways in Saccharomyces cerevisiae, Mol. Biol. Cell 12 (2001) 997-1007.

[18] M.K. Storey, K.L. Clay, T. Kutateladze, R.C. Murphy, M. Overduin, D.R. Voelker, Phosphatidylethanolamine has an essential role in Saccharomyces cerevisiae that is independent of its ability to form hexagonal phase structures, J. Biol. Chem. 276 (2001) 48539-48548.

[19] P. McGraw, S.A. Henry, Mutations in the Saccharomyces cerevisiae OPI3 gene: effects on phospholipid methylation, growth and cross-pathway regulation of inositol synthesis, Genetics 122 (1989) 317-330.

[20] H.A. Boumann, J. Gubbens, M.C. Koorengevel, C.S. Oh, C.E. Martin, A.J. Heck, J. Patton-Vogt, S.A. Henry, B. de Kruijff, A.I. de Kroon, Depletion of phosphatidylcholine in yeast induces shortening and increased saturation of the lipid acyl chains: evidence for regulation of intrinsic membrane curvature in a eukaryote, Mol. Biol. Cell 17 (2006) $1006-1017$.

[21] B. Gaigg, R. Simbeni, C. Hrastnik, F. Paltauf, G. Daum, Characterization of a microsomal subfraction associated with mitochondria of the yeast, Saccharomyces cerevisiae. Involvement in synthesis and import of phospholipids into mitochondria, Biochim. Biophys. Acta 1234 (1995) 214-220.

[22] E. Zinser, C.D. Sperka-Gottlieb, E.V. Fasch, S.D. Kohlwein, F. Paltauf, G. Daum, Phospholipid synthesis and lipid composition of subcellular membranes in the unicellular eukaryote Saccharomyces cerevisiae, J. Bacteriol. 173 (1991) 2026-2034.

[23] P.J. Trotter, D.R. Voelker, Identification of a non-mitochondrial phosphatidylserine decarboxylase activity (PSD2) in the yeast Saccharomyces cerevisiae, J. Biol. Chem. 270 (1995) 6062-6070.

[24] T. Kodaki, S. Yamashita, Yeast phosphatidylethanolamine methylation pathway. Cloning and characterization of two distinct methyltransferase genes, J. Biol. Chem. 262 (1987) 15428-15435.

[25] E.F. Summers, V.A. Letts, P. McGraw, S.A. Henry, Saccharomyces cerevisiae cho 2 mutants are deficient in phospholipid methylation and cross-pathway regulation of inositol synthesis, Genetics 120 (1988) 909-922.

[26] A.G. Howe, V. Zaremberg, C.R. McMaster, Cessation of growth to prevent cell death due to inhibition of phosphatidylcholine synthesis is impaired at 37 degrees C in Saccharomyces cerevisiae, J. Biol. Chem. 277 (2002) 44100-44107.

[27] C.R. McMaster, R.M. Bell, Phosphatidylcholine biosynthesis via the CDP-choline pathway in Saccharomyces cerevisiae. Multiple mechanisms of regulation, J. Biol. Chem. 269 (1994) 14776-14883.

[28] W.K. Huh, J.V. Falvo, L.C. Gerke, A.S. Carroll, R.W. Howson, J.S. Weissman, E.K. O'Shea, Global analysis of protein localization in budding yeast, Nature 425 (2003) 686-691.

[29] A. Leber, C. Hrastnik, G. Daum, Phospholipid-synthesizing enzymes in Golgi membranes of the yeast, Saccharomyces cerevisiae, FEBS Lett. 377 (1995) 271-274.

[30] G.M. Carman, Phosphatidate phosphatases and diacylglycerol pyrophosphate phosphatases in Saccharomyces cerevisiae and Escherichia coli, Biochim. Biophys. Acta 1348 (1997) 45-55.

[31] G.S. Han, W.I. Wu, G.M. Carman, The Saccharomyces cerevisiae Lipin homolog is a $\mathrm{Mg}^{2+}$-dependent phosphatidate phosphatase enzyme, J. Biol. Chem. 281 (2006) 9210-9218.

[32] J.S. Flick, J. Thorner, Genetic and biochemical characterization of a phosphatidylinositol-specific phospholipase C in Saccharomyces cerevisiae, Mol. Cell. Biol. 13 (1993) 5861-5876.

[33] K. Athenstaedt, G. Daum, YMR313c/TGL3 encodes a novel triacylglycerol lipase located in lipid particles of Saccharomyces cerevisiae, J. Biol. Chem. 278 (2003) 23317-23323.

[34] K. Funato, B. Vallee, H. Riezman, Biosynthesis and trafficking of sphingolipids in the yeast Saccharomyces cerevisiae, Biochemistry 41 (2002) 15105-15114. 
[35] K. Kim, K.H. Kim, M.K. Storey, D.R. Voelker, G.M. Carman, Isolation and characterization of the Saccharomyces cerevisiae EKI1 gene encoding ethanolamine kinase, J. Biol. Chem. 274 (1999) 14857-14866.

[36] R.H. Hjelmstad, R.M. Bell, sn-1,2-diacylglycerol choline- and ethanolaminephosphotransferases in Saccharomyces cerevisiae. Mixed micellar analysis of the CPT1 and EPT1 gene products, J. Biol. Chem. 266 (1991) 4357-4365.

[37] M. Kellis, B.W. Birren, E.S. Lander, Proof and evolutionary analysis of ancient genome duplication in the yeast Saccharomyces cerevisiae, Nature 428 (2004) 617-624.

[38] Y. Tsukagoshi, J. Nikawa, K. Hosaka, S. Yamashita, Expression in Escherichia coli of the Saccharomyces cerevisiae CCT gene encoding cholinephosphate cytidylyltransferase, J. Bacteriol. 173 (1991) 2134-2136.

[39] R. Min-Seok, Y. Kawamata, H. Nakamura, A. Ohta, M. Takagi, Isolation and characterization of ECT1 gene encoding CTP: phosphoethanolamine cytidylyltransferase of Saccharomyces cerevisiae, J. Biochem. (Tokyo) 120 (1996) 1040-1047.

[40] J. Nikawa, Y. Tsukagoshi, S. Yamashita, Cloning of a gene encoding choline transport in Saccharomyces cerevisiae, J. Bacteriol. 166 (1986) $328-330$.

[41] C.R. McMaster, R.M. Bell, Phosphatidylcholine biosynthesis in Saccharomyces cerevisiae. Regulatory insights from studies employing null and chimeric sn-1,2-diacylglycerol choline- and ethanolaminephosphotransferases, J. Biol. Chem. 269 (1994) 28010-28016.

[42] M. Bürgermeister, R. Birner-Grünberger, R. Nebauer, G. Daum, Contribution of different pathways to the supply of phosphatidylethanolamine and phosphatidylcholine to mitochondrial membranes of the yeast Saccharomyces cerevisiae, Biochim. Biophys. Acta 1686 (2004) 161-168.

[43] M.J. Janssen, M.C. Koorengevel, B. de Kruijff, A.I. de Kroon, Transbilayer movement of phosphatidylcholine in the mitochondrial outer membrane of Saccharomyces cerevisiae is rapid and bidirectional, Biochim. Biophys. Acta 1421 (1999) 64-76.

[44] R. Schneiter, B. Brügger, R. Sandhoff, G. Zellnig, A. Leber, M. Lampl, K. Athenstaedt, C. Hrastnik, S. Eder, G. Daum, F. Paltauf, F.T. Wiel, S.D. Kohlwein, Electrospray ionization tandem mass spectrometry (ESI-MS/ MS) analysis of the lipid molecular species composition of yeast subcellular membranes reveals acyl chain-based sorting/remodeling of distinct molecular species en route to the plasma membrane, J. Cell Biol. 146 (1999) 741-754.

[45] M. Bürgermeister, R. Birner-Grünberger, M. Heyn, G. Daum, Contribution of different biosynthetic pathways to species selectivity of aminoglycerophospholipids assembled into mitochondrial membranes of the yeast Saccharomyces cerevisiae, Biochim. Biophys. Acta 1686 (2004) 148-160.

[46] T. Kodaki, S. Yamashita, Characterization of the methyltransferases in the yeast phosphatidylethanolamine methylation pathway by selective gene disruption, Eur. J. Biochem. 185 (1989) 243-251.

[47] H.S. Choi, A. Sreenivas, G.S. Han, G.M. Carman, Regulation of phospholipid synthesis in the yeast ckil\{Delta\} ekil\{Delta\} mutant defective in the Kennedy pathway: the $\mathrm{CHOl}$-encoded phosphatidylserine synthase is regulated by mRNA stability, J. Biol. Chem. 279 (2004) 12081-12087.

[48] Z. Xie, M. Fang, V.A. Bankaitis, Evidence for an intrinsic toxicity of phosphatidylcholine to Sec14p-dependent protein transport from the yeast Golgi complex, Mol. Biol. Cell 12 (2001) 1117-1129.

[49] H.A. Boumann, M.J. Damen, C. Versluis, A.J. Heck, B. de Kruijff, A.I. de Kroon, The two biosynthetic routes leading to phosphatidylcholine in yeast produce different sets of molecular species. Evidence for lipid remodeling, Biochemistry 42 (2003) 3054-3059.

[50] C.J. DeLong, Y.J. Shen, M.J. Thomas, Z. Cui, Molecular distinction of phosphatidylcholine synthesis between the CDP-choline pathway and phosphatidylethanolamine methylation pathway, J. Biol. Chem. 274 (1999) 29683-29688.

[51] H.A. Boumann, P.T. Chin, A.J. Heck, B. DeKruijff, A.I. De Kroon, The yeast phospholipid $N$-methyltransferases catalyzing the synthesis of phosphatidylcholine preferentially convert di-C16:1 substrates both in vivo and in vitro, J. Biol. Chem. 279 (2004) 40314-40319.
[52] D.R. Voelker, Genetic analysis of intracellular aminoglycerophospholipid traffic, Biochem. Cell. Biol. 82 (2004) 156-169.

[53] P.J. Trotter, J. Pedretti, D.R. Voelker, Phosphatidylserine decarboxylase from Saccharomyces cerevisiae. Isolation of mutants, cloning of the gene, and creation of a null allele, J. Biol. Chem. 268 (1993) 21416-21424

[54] P.M. Gaynor, G.M. Carman, Phosphatidylethanolamine methyltransferase and phospholipid methyltransferase activities from Saccharomyces cerevisiae. Enzymological and kinetic properties, Biochim. Biophys. Acta 1045 (1990) 156-163.

[55] R.H. Hjelmstad, S.C. Morash, C.R. McMaster, R.M. Bell, Chimeric enzymes. Structure-function analysis of segments of $s n$-1,2-diacylglycerol choline- and ethanolaminephosphotransferases, J. Biol. Chem. 269 (1994) 20995-21002.

[56] H.A. Boumann, B. De Kruijff, A.J. Heck, A.I. De Kroon, The selective utilization of substrates in vivo by the phosphatidylethanolamine and phosphatidylcholine biosynthetic enzymes Ept1p and Cpt1p in yeast, FEBS Lett. 569 (2004) 173-177.

[57] A.L. Henneberry, T.A. Lagace, N.D. Ridgway, C.R. McMaster, Phosphatidylcholine synthesis influences the diacylglycerol homeostasis required for Sec14p-dependent Golgi function and cell growth, Mol. Biol. Cell 12 (2001) 511-520.

[58] M. Waksman, Y. Eli, M. Liscovitch, J.E. Gerst, Identification and characterization of a gene encoding phospholipase D activity in yeast, J. Biol. Chem. 271 (1996) 2361-2364.

[59] S.A. Rudge, A.J. Morris, J. Engebrecht, Relocalization of phospholipase D activity mediates membrane formation during meiosis, J. Cell Biol. 140 (1998) 81-90.

[60] A. Sreenivas, J.L. Patton-Vogt, V. Bruno, P. Griac, S.A. Henry, A role for phospholipase D (Pld1p) in growth, secretion, and regulation of membrane lipid synthesis in yeast, J. Biol. Chem. 273 (1998) $16635-16638$

[61] Z. Xie, M. Fang, M.P. Rivas, A.J. Faulkner, P.C. Sternweis, J.A. Engebrecht, V.A. Bankaitis, Phospholipase D activity is required for suppression of yeast phosphatidylinositol transfer protein defects, Proc. Natl. Acad. Sci. U. S. A. 95 (1998) 12346-12351.

[62] J.A. Mayr, S.D. Kohlwein, F. Paltauf, Identification of a novel, $\mathrm{Ca}(2+)-$ dependent phospholipase D with preference for phosphatidylserine and phosphatidylethanolamine in Saccharomyces cerevisiae, FEBS Lett. 393 (1996) 236-240.

[63] M. Waksman, X. Tang, Y. Eli, J.E. Gerst, M. Liscovitch, Identification of a novel $\mathrm{Ca}^{2+}$-dependent, phosphatidylethanolamine-hydrolyzing phospholipase D in yeast bearing a disruption in PLD1, J. Biol. Chem. 272 (1997) $36-39$.

[64] K.S. Lee, J.L. Patton, M. Fido, L.K. Hines, S.D. Kohlwein, F. Paltauf, S.A. Henry, D.E. Levin, The Saccharomyces cerevisiae PLB1 gene encodes a protein required for lysophospholipase and phospholipase B activity, J. Biol. Chem. 269 (1994) 19725-19730.

[65] H. Fyrst, B. Oskouian, F.A. Kuypers, J.D. Saba, The PLB2 gene of Saccharomyces cerevisiae confers resistance to lysophosphatidylcholine and encodes a phospholipase B/lysophospholipase, Biochemistry 38 (1999) $5864-5871$.

[66] O. Merkel, M. Fido, J.A. Mayr, H. Pruger, F. Raab, G. Zandonella, S.D. Kohlwein, F. Paltauf, Characterization and function in vivo of two novel phospholipases B/lysophospholipases from Saccharomyces cerevisiae, J. Biol. Chem. 274 (1999) 28121-28127.

[67] O. Zaccheo, D. Dinsdale, P.A. Meacock, P. Glynn, Neuropathy target esterase and its yeast homologue degrade phosphatidylcholine to glycerophosphocholine in living cells, J. Biol. Chem. 279 (2004) 24024-24033.

[68] S.R. Dowd, M.E. Bier, J.L. Patton-Vogt, Turnover of Phosphatidylcholine in Saccharomyces cerevisiae. The role of the CDP-choline pathway, J. Biol. Chem. 276 (2001) 3756-3763.

[69] V. Zaremberg, C.R. McMaster, Differential partitioning of lipids metabolized by separate yeast glycerol-3-phosphate acyltransferases reveals that phospholipase D generation of phosphatidic acid mediates sensitivity to choline-containing lysolipids and drugs, J. Biol. Chem. 277 (2002) 39035-39044. 
[70] E. Fisher, C. Almaguer, R. Holic, P. Griac, J. Patton-Vogt, Glycerophosphocholine-dependent growth requires Gde1p (YPL110c) and Gitlp in Saccharomyces cerevisiae, J. Biol. Chem. 280 (2005) 36110-36117.

[71] J.P. Fernandez-Murray, C.R. McMaster, Glycerophosphocholine catabolism as a new route for choline formation for phosphatidylcholine synthesis by the Kennedy pathway, J. Biol. Chem. 280 (2005) 38290-38296.

[72] A. Yamashita, T. Sugiura, K. Waku, Acyltransferases and transacylases involved in fatty acid remodeling of phospholipids and metabolism of bioactive lipids in mammalian cells, J. Biochem. (Tokyo) 122 (1997) $1-16$.

[73] R.W. Yost, S.J. Grauvickel, R. Cantwell, J.S. Bomalaski, A.P. Hudson, Yeast mitochondria (Saccharomyces cerevisiae) contain $\mathrm{Ca}^{2+}$-independent phospholipase A1 and A2 activities: effect of respiratory state, Biochem. Int. 24 (1991) 199-208.

[74] P.C. Bradshaw, D.W. Jung, D.R. Pfeiffer, Free fatty acids activate a vigorous $\mathrm{Ca}^{2+}: 2 \mathrm{H}^{+}$antiport activity in yeast mitochondria, J. Biol. Chem. 276 (2001) 40502-40509.

[75] A. Dahlqvist, U. Stahl, M. Lenman, A. Banas, M. Lee, L. Sandager, H. Ronne, S. Stymne, Phospholipid:diacylglycerol acyltransferase: an enzyme that catalyzes the acyl-CoA-independent formation of triacylglycerol in yeast and plants, Proc. Natl. Acad. Sci. U. S. A. 97 (2000) 6487-6492.

[76] P. Oelkers, A. Tinkelenberg, N. Erdeniz, D. Cromley, J.T. Billheimer, S.L. Sturley, A lecithin cholesterol acyltransferase-like gene mediates diacylglycerol esterification in yeast, J. Biol. Chem. 275 (2000) 15609-15612.

[77] M.G. Richard, C.R. McMaster, Lysophosphatidylcholine acyltransferase activity in Saccharomyces cerevisiae: regulation by a high-affinity $\mathrm{Zn} 2$ +binding site, Lipids 33 (1998) 1229-1234.

[78] K. Brandner, D.U. Mick, A.E. Frazier, R.D. Taylor, C. Meisinger, P. Rehling, Taz1, An outer mitochondrial membrane protein, affects stability and assembly of inner membrane protein complexes: implications for Barth Syndrome, Mol. Biol. Cell 16 (2005) 5202-5214.

[79] Z. Gu, F. Valianpour, S. Chen, F.M. Vaz, G.A. Hakkaart, R.J. Wanders, M. L. Greenberg, Aberrant cardiolipin metabolism in the yeast taz1 mutant: a model for Barth syndrome, Mol. Microbiol. 51 (2004) 149-158.

[80] E. Testet, J. Laroche-Traineau, A. Noubhani, D. Coulon, O. Bunoust, N. Camougrand, S. Manon, R. Lessire, J.J. Bessoule, Ypr140wp, 'the yeast tafazzin', displays a mitochondrial lysophosphatidylcholine (lyso-PC) acyltransferase activity related to triacylglycerol and mitochondrial lipid synthesis, Biochem. J. 387 (2005) 617-626.

[81] S.M. Gruner, Intrinsic curvature hypothesis for biomembrane lipid composition: a role for nonbilayer lipids, Proc. Natl. Acad. Sci. U. S. A. 82 (1985) 3665-3669.
[82] E. van den Brink-van der Laan, J.A. Killian, B. de Kruijff, Nonbilayer lipids affect peripheral and integral membrane proteins via changes in the lateral pressure profile, Biochim. Biophys. Acta 1666 (2004) 275-288.

[83] V.M. Gohil, M.N. Thompson, M.L. Greenberg, Synthetic lethal interaction of the mitochondrial phosphatidylethanolamine and cardiolipin biosynthetic pathways in Saccharomyces cerevisiae, J. Biol. Chem. 280 (2005) 35410-35416.

[84] V.A. Bankaitis, A.J. Morris, Lipids and the exocytotic machinery of eukaryotic cells, Curr. Opin. Cell Biol. 15 (2003) 389-395.

[85] Y. Jun, R.A. Fratti, W. Wickner, Diacylglycerol and its formation by phospholipase $\mathrm{C}$ regulate Rab- and SNARE-dependent yeast vacuole fusion, J. Biol. Chem. 279 (2004) 53186-53195.

[86] R. Koynova, M. Caffrey, Phases and phase transitions of the hydrated phosphatidylethanolamines, Chem. Phys. Lipids 69 (1994) 1-34.

[87] H. Okuyama, M. Saito, V.C. Joshi, S. Gunsberg, S.J. Wakil, Regulation by temperature of the chain length of fatty acids in yeast, J. Biol. Chem. 254 (1979) 12281-12284.

[88] M. Suutari, K. Liukkonen, S. Laakso, Temperature adaptation in yeasts: the role of fatty acids, J. Gen. Microbiol. 136 (1990) 1469-1474.

[89] W. Dowhan, Molecular basis for membrane phospholipid diversity: why are there so many lipids? Annu. Rev. Biochem. 66 (1997) 199-232.

[90] R. Schneiter, V. Tatzer, G. Gogg, E. Leitner, S.D. Kohlwein, Elo1pdependent carboxy-terminal elongation of C14:1Delta(9) to C16:1Delta (11) fatty acids in Saccharomyces cerevisiae, J. Bacteriol. 182 (2000) $3655-3660$.

[91] P. Kaliszewski, T. Ferreira, B. Gajewska, A. Szkopinska, T. Berges, T Zoladek, Enhanced levels of Pis1p (phosphatidylinositol synthase) improve the growth of Saccharomyces cerevisiae cells deficient in Rsp5 ubiquitin ligase, Biochem. J. 395 (2006) 173-181.

[92] R. Nayar, S.L. Schmid, M.J. Hope, P.R. Cullis, Structural preferences of phosphatidylinositol and phosphatidylinositol-phosphatidylethanolamine model membranes. Influence of $\mathrm{Ca}^{2+}$ and $\mathrm{Mg}^{2+}$, Biochim. Biophys. Acta 688 (1982) 169-176.

[93] J. Gagne, L. Stamatatos, T. Diacovo, S.W. Hui, P.L. Yeagle, J.R. Silvius, Physical properties and surface interactions of bilayer membranes containing N-methylated phosphatidylethanolamines, Biochemistry 24 (1985) 4400-4408

[94] J.Y. Choi, W.E. Martin, R.C. Murphy, D.R. Voelker, Phosphatidylcholine and N-methylated phospholipids are non-essential in Saccharomyces cerevisiae, J. Biol. Chem. 279 (2004) 42321-42330.

[95] S.A. Henry, J.L. Patton-Vogt, Genetic regulation of phospholipid metabolism: yeast as a model eukaryote, Prog. Nucleic Acid Res. Mol. Biol. 61 (1998) 133-179. 